INPLASY

PROTOCOL

To cite: Liang et al. The Effect of Triptolide in Animal Models for Diabetic Nephropathy: A

Systematic Review and Metaanalysis. Inplasy protocol

2020100042. doi:

10.37766/inplasy2020.10.0042

Received: 11 October 2020

Published: 12 October 2020

Corresponding author:

Dongning Liang

Idning1@163.com

Author Affiliation:

The first clinical medical college of Southern Medical University

Support: No funding.

Review Stage at time of this submission: The review has not yet started.

Conflicts of interest:

No conflicts of interest exist.

\section{The Effect of Triptolide in Animal Models for Diabetic Nephropathy: A Systematic Review and Meta-analysis}

\author{
Liang, D1; Mai, H²; Ruan, F3.
}

Review question / Objective: What are the effect of triptolide in animal models for diabetic nephropathy?

Condition being studied: A doubtless public health problem caused by diabetes ensued. An epidemiology investigation, conducted by Lin Li between 2002 to 2013, the prevalence of diabetes increased at different rates. Such a phenomenon happens in other parts of the world. Another investigation conducted by Weiping Teng, indicates that the prevalence of diabetes has progressively increased among adults from 2007 to 2017. As a common but complex implication of diabetes, diabetic nephropathy deserves much attention for being the main cause of end-stage renal failure. Since the clinical recommended therapies like ACEI or ARB have brought great financial pressure to the patients, new drugs should be developed. Chinese herbal medicine has been widely used in East Asia for illness treatment. In existing historical literature, Tripterygium wilfordii has frequently used for diabetic nephropathy. With the development of purification of herbal medicine, it is possible to identify its pharmacological activities. One constituent of Tripterygium wilfordii, triptolide has been tested in many animal experiments to see whether it could work in curing such a disease. This systematic review aims to evaluate the efficacy of triptolide rigorously as an alternative therapy for diabetic nephropathy.

INPLASY registration number: This protocol was registered with the International Platform of Registered Systematic Review and Meta-Analysis Protocols (INPLASY) on 12 October 2020 and was last updated on 12 October 2020 (registration number INPLASY2020100042).

\section{INTRODUCTION}

Review question / Objective: What are the effect of triptolide in animal models for diabetic nephropathy?
Condition being studied: A doubtless public health problem caused by diabetes ensued. An epidemiology investigation, conducted by $\mathrm{Lin} \mathrm{Li}$ between 2002 to 2013, the prevalence of diabetes increased at 
different rates. Such a phenomenon happens in other parts of the world. Another investigation conducted by Weiping Teng, indicates that the prevalence of diabetes has progressively increased among adults from 2007 to 2017. As a common but complex implication of diabetes, diabetic nephropathy deserves much attention for being the main cause of end-stage renal failure. Since the clinical recommended therapies like ACEI or ARB have brought great financial pressure to the patients, new drugs should be developed. Chinese herbal medicine has been widely used in East Asia for illness treatment. In existing historical literature, Tripterygium wilfordii has frequently used for diabetic nephropathy. With the development of purification of herbal medicine, it is possible to identify its pharmacological activities. One constituent of Tripterygium wilfordii, triptolide has been tested in many animal experiments to see whether it could work in curing such a disease. This systematic review aims to evaluate the efficacy of triptolide rigorously as an alternative therapy for diabetic nephropathy.

\section{METHODS}

Search strategy: The study only selects RCTs of triptolide treatment for animal models of diabetic nephropathy. We will search 4 English database including the Cochrane Library, Pubmed, Embase, Web of science and 4 Chinese database including China National Knowledge Infrastructure(CNKI), Wanfang Database, Chinese Biomedical Database, Chinese Science and Technology Journal Database from the built-in to October 2020. We will also include the eligible reference of included publications.

Participant or population: Animal models of diabetic nephropathy.

Intervention: Triptolide treatment only.

Comparator: Recommended therapies, including ACEI and ARB, or equal volumes of nonfunctional liquid (normal saline) or no treatment.
Study designs to be included: Randomized controlled studies.

Eligibility criteria: Included criteria: 1. All animal models with diabetic nephropathy, regardless of species, ages or sexes. 2 . The experimental groups used triptolide as monotherapy in any dose, while the control groups received equal volumes of nonfunctional liquid (normal saline) or no treatment or recommended therapies according to clinical practice guidelines, including ACEi or ARB. 3. Literature is either published in Chinese or English. 4. Triptolide preparation in various forms, including extracts, granules, and injections, were eligible. 5. The outcomes were change in albuminuria and proteinuria excretion, change in kidney function, including $\mathrm{SCr}$ and BUN. Excluded criteria: 1. Not in vivo studies (in vitro studies, clinical trials, review articles, case reports, comments, editorials, and abstracts) 2 . Additional hypoglycemics were used during the course of treatment. 3. Assessing triptolide combined with other herbal ingredients or complementary therapies. 4. Repeated literatures. 5. Full-text not found.

Information sources: We will select the studies from eight databases, including the Cochrane Library, PubMed, Embase, Web of science, China National Knowledge Infrastructure(CNKI), Wanfang Database, Chinese Biomedical Database, Chinese Science and Technology Journal Database. The eligible references will also be included.

Main outcome(s): Main outcomes include albuminuria, proteinuria excretion, changes in kidney function including $\mathrm{SCr}$ and BUN.

Quality assessment / Risk of bias analysis: The Systematic Review Centre for Laboratory Animal Experimentation Risk of Bias toll will be applied to evaluate the risk of bias in individual included studies. Each eligible study will be assessed by two reviewers independently. The discrepancies will be solved by discussion. According to the SYRCLE's tool, Studies will be divided into low risk, high risk and unclear. 
Strategy of data synthesis: RevMan5.3 software downloaded from Cochrane Collaboration will be adopted to analyze data. Continuous variables will be expressed as mean difference (MD) or standardized mean difference (SMD) with $95 \%$ confidence interval $(95 \% \mathrm{CI})$. Heterogeneity among included studies will be checked by using chi-square test and 12 test. We will use a random-effects model in meta-analysis if there are $12>50 \%$ and $p<$ 0.10 . Otherwise, a fixed-effects model will be chosen.

Subgroup analysis: Subgroup analysis will be carried out by comparisons.

Sensibility analysis: We will remove lowrisk studies one by one from the group of synthesized studies, and re-analyze the rest to estimate the robustness of results.

Country(ies) involved: China.

Keywords: Triptolide, diabetic nephropathy, animal models, systematic review.

Contributions of each author:

Author 1 - Dongning Liang.

Author 2 - Hanwen Mai.

Author 3 - Fangyi Ruan. 